\title{
Benign Melanosis as the Cause of Diffuse Black Pigmentation in Prostate Resection
}

\author{
Basri Cakiroglu ${ }^{\mathrm{a}, \mathrm{e}}$, Seyit Erkan Eyyupoglu ${ }^{\mathrm{b}}$, Orhun Sinanoglu ${ }^{\mathrm{c}}$, Bora Gurel ${ }^{\mathrm{d}}$
}

\begin{abstract}
Macroscopically, black pigmentation of the prostate tissue is a very rare condition in urological surgery. This paper reviews melanotic pigmentation as the pathological cause of black prostatic discoloration encountered during transurethral resection performed upon diagnosis of benign prostatic hypertrophy and discusses it within the frame of the differential diagnosis.
\end{abstract}

Keywords: Melanosis; Prostate; Benign prostate hyperplasia; Transurethral resection

\section{Introduction}

Black prostate tissues, although very rarely, are encountered in pathology laboratories rather than the inherently white prostate tissues [1-4]. Macroscopic black discoloration can occur due to intense deposition of the tissue with hemosiderin, homogentisic acid, lipofuscin or melanin pigments [1, 2]. There are no literature reports showing the deposition of lipofuscin to an extent where the tissue has a black macroscopic appearance. Melanin deposition in the prostate tissue is a very rare condition except for melanoma and was first reported in the literature as blue nevus, but later defined as

Manuscript accepted for publication October 10, 2013

${ }^{a}$ Hisar Intercontinental Hospital, Deparment of Urology, Saray Mah. Siteyolu Cad. No. 7, 34768 Umraniye, Istanbul, Turkey

${ }^{\mathrm{b}}$ Sabuncuoglu Serafettin Amasya Teaching and Research Hospital, Department of Urology, Amasya, Turkey

${ }^{c}$ Maltepe University Medical School Department of Urology, Istanbul, Turkey

${ }^{\mathrm{d} S a b u n c u o g l u}$ Serafettin Amasya Teaching and Research Hospital, Department of Pathology, Amasya, Turkey

${ }^{\mathrm{e}}$ Corresponding author: Basri Cakiroglu, Hisar Intercontinental Hospital, Department of Urology, Saray Mah.Siteyolu Cad. No. 7, 34768

Umraniye, Istanbul, Turkey. Email: drbasri@gmail.com

doi: http://dx.doi.org/10.4021/wjnu117w melanosis due to a different histological pattern. Blue nevi of the prostate have been reported in 20 cases in the literature [1-10]. However, prostatic melanosis is very rare, with about nine cases reported $[6,8]$.

In this paper, we presented a prostatic melanosis pathologically found due to the extensive black-gray pigmentation macroscopically seen in the transurethral prostate resection of an 82-year-old patient, and reviewed the literature.

\section{Case Report}

Urological examination of an 82-year-old patient who was admitted with complaints of the lower urinary system revealed prostatic enlargement in digital rectal examination with a consistency of $(++)$ fibroadenoma, with no nodules and induration that would verify tumor growth. The ultrasound examination showed no echo pattern abnormality with prostatic size of about $55 \mathrm{~g}$. The post-voiding residual urine volume was $110 \mathrm{~mL}$. The International Prostate Symptom Score was 21. Total prostatic specific antigen (PSA) value was $2.27 \mathrm{ng} / \mathrm{mL}$. The patient, who was not satisfied with his quality of life despite medical treatment, therefore underwent transurethral prostate resection surgery.

No pathologies were found in other preoperative system examinations and routine analyses. The patient's postoperative prostatic urethral epithelium was not as bright red as normal ones. The epithelium was more opaque and dark purple in color. Following initial epithelial resections, a grayblackish color was observed instead of the white color of the prostate tissue. Even the bleedings during the resections did not change this ash-gray color of the tissue. The white color was visible when the capsule was reached.

Clinical data and perioperative macroscopic findings were noted and the specimen was sent to the pathology laboratory. Pathological examination found melanin pigmentation both in the epithelial tissue and the stromal tissue next to the fibroepithelial hyperplasia, with a diagnosis of prostatic melanosis. No epithelial, stromal, and melanocytic malignancies were found (Fig. 1).

Dermatological and other system examinations during the postoperative period revealed no other melanocytic dis- 

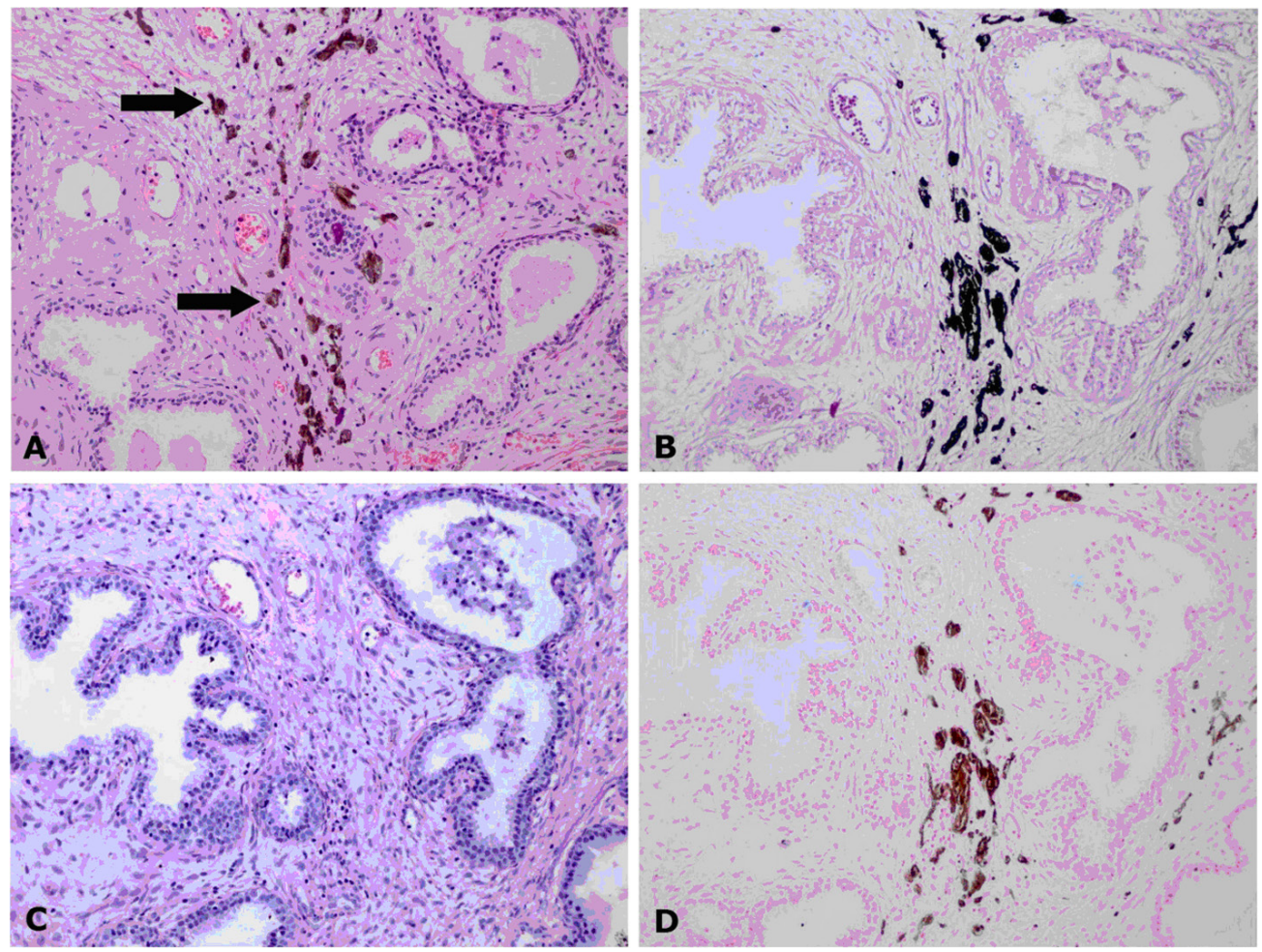

Figure 1. (A-D) Pathological examination found melanin pigmentation both in the epithelial tissue and the stromal tissue next to the fibroepithelial hyperplasia, with a diagnosis of prostatic melanosis. No epithelial, stromal, and melanocytic malignancies were found.

eases. The patient was followed for 2 years postoperatively with periodical tests and examinations. PSA and uroflowmetry values were normal.

\section{Discussion}

The melanocytic activity in the prostate was coincidentally found and first documented by Nigogosyan and co-workers during an autopsy in 1963 [5]. This paper was quoted in almost all subsequent case studies. Melanotic lesions of the prostate were divided into three groups in order to avoid the confusion in the literature: 1) melanosis, 2) blue nevus, and 3) malignant melanoma [1-5]. Nigogosyan found the accumulations of melanin in the fibromuscular stroma of the prostate [5]. This was likened to dermal blue nevus, which is comparable to it, and was named the blue nevus of the prostate [5] and in melanosis, there is melanin deposition both in the stroma and the acinar epithelium. Both of them are benign conditions and no malignancies were reported to have developed from these underlying conditions $[1,2,9]$.

The age distribution range of this rare benign entity reported in the literature is 21-86, with a mean age of 66 [1-4]. One can conclude that it is more common among Caucasians considering the cases reported in the USA, Israel, Spain, India, Italy, Brazil, Libya, Romania, and Greece and that it is prevalent in various regions of the world $[4,5,7,9]$. Our patient was an 82-year-old Caucasian, and as this was the first case reported in Turkey, a literature review shows that the cases are patients who were admitted with chronic lower urinary tract complaints [7]. Benign prostatic melanin depositions with no specific symptoms were found incidentally by the pathology laboratory in the transrectal biopsy material as a result of raised PSA, open surgery as a result of benign prostatic hypertrophy, in transurethral resection chips, or in non-cancerous regions in radical prostatectomy made due to prostate cancer and in well-differentiated adenocarcinoma cells $[1,6,8,9]$. In our case, the prostate tissue had a relatively black and grayish appearance and its pathology was identified as melanosis. Histopathological diagnosis is based on the sight of melanin deposition in the prostate tissue. The sections are stained with hematoxylin and eosin (HE) during the routine histological evaluation of the prostate blocks. Melanin looks dark brownish in HE preparations. To verify the presence of melanin, they are stained with the FontanaMasson (FM) technique to make the pigments look black, and then lost non-melanin pigmentations are sought with melanin bleaching. Melanoma exhibits a black color in the prostate and contains cells that are obviously histologically malignant in appearance; histological malignity criteria including atypia, pleomorphism, distortion of the architecture, increased mitotic activity, and poor differentiation are actu- 
ally important. In addition to histological evaluations, immunohistochemical (IHC) staining is also based on the S100 and HMB45 antidote. S100 negativity and HMB45 positivity are sought in malignant melanoma. In case of doubt, its reverse by IHC staining helps the diagnosis of benign lesions $[2,7,9]$. In our case, the presence of suspected melanin intensively in the stroma and partly in the epithelium stained positive with FM in addition to the fibroepithelial hyperplasia identified by HE staining, and was confirmed with melanin bleaching method. IHC examination with the HMB45 antidote was negative despite the lack of cellular atypia. The importance of benign lesions for pathology is their differentiation from malign lesions and the over diagnosis during the diagnosis stage. Other causes of prostatic black discoloration are lipofuscin and hemosiderin, which may be confused with melanin $[1,2]$. While hemosiderin has large golden-brown grains and is generally in the stromal macrophages, lipofuscin has fine yellowish-brown grains. Differentiation may be made with Ziehl-Neelsen, periodic acid-Schiff, and Prussian blue [10].

\section{Conclusion}

The presentation of our case will strengthen the literature suggesting that black-ash-colored prostate resection may be encountered in urological surgery in cases with such intensive deposition.

\section{Conflict of Interest}

None declared.

\section{References}

1. Redondo Martinez E, Rey Lopez A, Diaz Cascajo C. [Blue nevus of the prostate. Differential diagnosis of prostatic pigmented lesions]. Arch Esp Urol. 1998;51(3):286-289.

2. Dailey VL, Hameed O. Blue nevus of the prostate. Arch Pathol Lab Med. 2011;135(6):799-802.

3. Langley JW, Weitzner S. Blue nevus and melanosis of prostate. J Urol. 1974;112(3):359-361.

4. Kovi J, Jackson AG, Jackson MA. Blue nevus of the prostate: ultrastructural study. Urology. 1977;9(5):576578.

5. Nigogosyan G, Delapava S, Pickren JW, Woodruff MM. Blue Nevus of the Prostate Gland. Cancer. 1963;16:10971099.

6. Furusato M, Matsumoto I, Kato H, Takahashi H, Kawashima Y, Wada T, Suzuki M, et al. Prostatic carcinoma with melanosis. Prostate. 1989;15(1):65-69.

7. Martinez Martinez CJ, Garcia Gonzalez R, Castaneda Casanova AL. Blue nevus of the prostate: report of two new cases with immunohistochemical and electron-microscopic studies. Eur Urol. 1992;22(4):339-342.

8. Klock C, Gomes R, Joao M, Netto G. Prostate melanosis associated with acinar adenocarcinoma. Int J Surg Pathol. 2010;18(5):379-380.

9. Anderco D, Lazar E, Taban S, Miclea F, Dema A. Prostatic blue nevus. Rom J Morphol Embryol. 2010;51(3):555-557.

10. Aguilar M, Gaffney EF, Finnerty DP. Prostatic melanosis with involvement of benign and malignant epithelium. J Urol. 1982;128(4):825-827. 